\title{
Apelin-13/APJ system attenuates early brain injury via suppression of endoplasmic reticulum stress-associated TXNIP/NLRP3 inflammasome activation and oxidative stress in a AMPK-dependent manner after subarachnoid hemorrhage in rats
}

Weilin $\mathrm{Xu}^{1 \dagger}$, Tao $\mathrm{Li}^{1 \dagger}$, Liansheng Gao ${ }^{1 \dagger}$, Jingwei Zheng ${ }^{1}$, Jun Yan², Jianmin Zhang ${ }^{1,3,4^{*}}$ and Anwen Shao ${ }^{1 *}$ (D)

\begin{abstract}
Background: Neuroinflammation and oxidative stress play important roles in early brain injury following subarachnoid hemorrhage (SAH). This study is the first to show that activation of apelin receptor (APJ) by apelin-13 could reduce endoplasmic reticulum (ER)-stress-associated inflammation and oxidative stress after SAH.

Methods: Apelin-13, apelin siRNA, APJ siRNA, and adenosine monophosphate-activated protein kinase (AMPK) inhibitor-dorsomorphin were used to investigate if the activation of APJ could provide neuroprotective effects after $\mathrm{SAH}$. Brain water content, neurological functions, blood-brain barrier (BBB) integrity, and inflammatory molecules were evaluated at $24 \mathrm{~h}$ after SAH. Western blotting and immunofluorescence staining were applied to assess the expression of target proteins.

Results: The results showed that endogenous apelin, APJ, and p-AMPK levels were significantly increased and peaked in the brain $24 \mathrm{~h}$ after SAH. In addition, administration of exogenous apelin-13 significantly alleviated neurological functions, attenuated brain edema, preserved BBB integrity, and also improved long-term spatial learning and memory abilities after SAH. The underlying mechanism of the neuroprotective effects of apelin-13 is that it suppresses microglia activation, prevents ER stress from overactivation, and reduces the levels of thioredoxininteracting protein (TXNIP), NOD-like receptor pyrin domain-containing 3 protein (NLRP3), Bip, cleaved caspase-1, IL$1 \beta$, TNFa, myeloperoxidase (MPO), and reactive oxygen species (ROS). Furthermore, the use of APJ siRNA and dorsomorphin abolished the neuroprotective effects of apelin-13 on neuroinflammation and oxidative stress.
\end{abstract}

Conclusions: Exogenous apelin-13 binding to APJ attenuates early brain injury by reducing ER stress-mediated oxidative stress and neuroinflammation, which is at least partly mediated by the AMPK/TXNIP/NLRP3 signaling pathway.

Keywords: Early brain injury, SAH, Neuroinflammation, Oxidative stress, Apelin-13, APJ, NLRP3

\footnotetext{
* Correspondence: zjm135@zju.edu.cn; 21118116@zju.edu.cn

${ }^{\dagger}$ Weilin Xu, Tao Li and Liansheng Gao contributed equally to this work.

'Department of Neurosurgery, Second Affiliated Hospital, School of Medicine,

Zhejiang University, 88 Jiefang Rd, Hangzhou 310009, Zhejiang, China

Full list of author information is available at the end of the article
}

(C) The Author(s). 2019 Open Access This article is distributed under the terms of the Creative Commons Attribution 4.0 International License (http://creativecommons.org/licenses/by/4.0/), which permits unrestricted use, distribution, and

reproduction in any medium, provided you give appropriate credit to the original author(s) and the source, provide a link to the Creative Commons license, and indicate if changes were made. The Creative Commons Public Domain Dedication waiver (http://creativecommons.org/publicdomain/zero/1.0/) applies to the data made available in this article, unless otherwise stated. 


\section{Background}

Early brain injury (EBI), which is induced within 3 days after subarachnoid hemorrhage (SAH), was reported to be the main cause of poor prognosis of patients with $\mathrm{SAH}[8,31]$. The underlying mechanisms include neuronal apoptosis, neuroinflammation, oxidative stress, and blood-brain barrier disruption [6, 46]. Increasing studies support the idea that ER stress plays a major role in the post-SAH pathophysiological process $[15,44,48]$. The over activation of ER stress induces calcium release and oxidative stress, which further trigger downstream cascading reactions resulting in inflammation and cellular apoptosis [53]. Li et al. showed that the activation of the thioredoxin-interacting protein (TXNIP)/NOD-like receptor pyrin domain-containing 3 protein (NLRP3) inflammasome could link ER stress to inflammation and cellular apoptosis in brain tissues $[16,17,19]$.

Apelin, a peptide with 77 amino acids, is an endogenous ligand for the G protein-coupled receptor APJ [24]. It can be degraded into four shorter active forms, including apelin-12, apelin-13, apelin-17, and apelin-36 ([14] [30];). Among those short forms, apelin-13 displays the strongest biological activity [32, 40]. Recently, apelin-13 has received significant attention from neurologists owing to its neuroprotective effects in the central nervous system. Apelin-13 has displayed wide bioactivity via its receptor APJ, including anti-oxidative stress, antiapoptosis, and anti-inflammation [26, 40]. However, the role of APJ has not been explored in the SAH rat model.

In addition, $\mathrm{Wu}$ et al. showed that apelin-13 could attenuate ER stress-mediated neuronal apoptosis in ischemic stroke [36]. Apelin-13 exerts its neuroprotective effects via its receptor APJ, which further activates adenosine monophosphate-activated protein kinase (AMPK) or suppresses the activation of NLRP3 [12, 43]. In addition, the activation of AMPK leads to phosphorylation and degradation of TXNIP, which could further suppress the overactivation of ER stress and reduce the level of NLRP3 $[16,17,19]$. However, the mechanisms underlying how apelin-13/APJ regulates ER stress and suppresses the activation of NLRP3 after SAH are still unclear.

In this study, we wanted to verify the following hypotheses: (1) SAH could result in the increase of apelin13, APJ, and p-AMPK levels; (2) apelin-13 could reduce brain edema, BBB disruption, and neurofunctional deficits; (3) knockdown of APJ with siRNA could aggravate neuroinflammation; and (4) selective inhibition of pAMPK with dorsomorphin could reverse the antineuroinflammatory effects of apelin-13.

\section{Methods}

\section{Animals}

All animal experiments were performed according to the Institutional Animal Care and Use Committee of
Zhejiang University. The procedures were conducted according to the National Institutes of Health's Guide for the Care and the Use of Laboratory Animals and the ARRIVE (Animal Research: Reporting In Vivo Experiments) guidelines. We used Sprague-Dawley (SD, male) rats (280-330 g) (SLAC Laboratory Animal Co., Ltd. Shanghai, China) in this study. We kept the rats in a 12$\mathrm{h}$ day/night cycle $\left(22 \pm 1{ }^{\circ} \mathrm{C} ; 60 \pm 5 \%\right.$ humidity). The rats had free access to water and food.

\section{Experimental design}

This study included five separate experiments using a rat model of SAH (Fig. 1). A total of 312 rats were used in this study, including shared, dead, and excluded animals among each group.

In the first step, the expression of endogenous apelin13 , its receptor APJ, and p-AMPK protein levels were assessed in the sham group and at different time points in the SAH groups. Rats were randomly distributed into seven groups: sham, SAH $3 \mathrm{~h}, \mathrm{SAH} 6 \mathrm{~h}, \mathrm{SAH} 12 \mathrm{~h}, \mathrm{SAH}$ $24 \mathrm{~h}, \mathrm{SAH} 48 \mathrm{~h}$, and SAH $72 \mathrm{~h}$. The ipsilateral/left cerebral cortex from each group was collected for Western blot analysis. The cellular localization of APJ was detected using double immunofluorescence staining (sham and $24 \mathrm{~h}$ SAH groups).

In the second step, to study the neuroprotective effects of apelin-13, rats were randomly distributed into sham, $\mathrm{SAH}+$ vehicle $(10 \mu \mathrm{l}$ sterile saline $), \mathrm{SAH}+$ apelin-13 $(15 \mu \mathrm{g} / \mathrm{kg}), \mathrm{SAH}+$ apelin-13 $(50 \mu \mathrm{g} / \mathrm{kg})$, and $\mathrm{SAH}+$ apelin-13 $(150 \mu \mathrm{g} / \mathrm{kg})$. Apelin-13 was intracerebroventricularly given $30 \mathrm{~min}$ after $\mathrm{SAH}$. The rats in the sham group received the same procedures as the $\mathrm{SAH}+\mathrm{ve}-$ hicle group except injection. Neurological score, brain water content, and Evans blue leakage were measured $24 \mathrm{~h}$ after $\mathrm{SAH}$ in all groups.

In the third step, to explore the effects of treatment with apelin-13 $(50 \mu \mathrm{g} / \mathrm{kg}$, Santa Cruz, sc-351718) on long-term spatial learning and memory, rats were separated into three groups: sham, $\mathrm{SAH}+$ vehicle, and $\mathrm{SAH}+$ apelin-13. Morris water maze was conducted on days 21-25 after SAH.

In the fourth step, we adopted APJ siRNA to further evaluate the role of APJ on the anti-neuroinflammatory effects of exogenous apelin-13. Rats were randomly distributed into sham, SAH + vehicle, SAH + apelin-13 $(50 \mu \mathrm{g} / \mathrm{kg}), \mathrm{SAH}+$ apelin-13 + scramble siRNA $(500 \mathrm{pmol}$ in $10 \mu \mathrm{l}$ in sterile saline), and $\mathrm{SAH}+$ apelin-13 + APJ siRNA (500 pmol in $10 \mu \mathrm{l}$ in sterile saline). APJ siRNA was injected intracerebroventricularly at $48 \mathrm{~h}$ before the induction of SAH. The rats in the sham group received the same procedures as $\mathrm{SAH}+$ vehicle group except for injection. The ipsilateral/left cerebral cortex from each group was sampled for Western blot analysis, ROS, and immunofluorescence staining $24 \mathrm{~h}$ after SAH. 
Experiment 1. Time-Course of apelin-13, APJ, and p-AMPK, cellular localization of APJ.

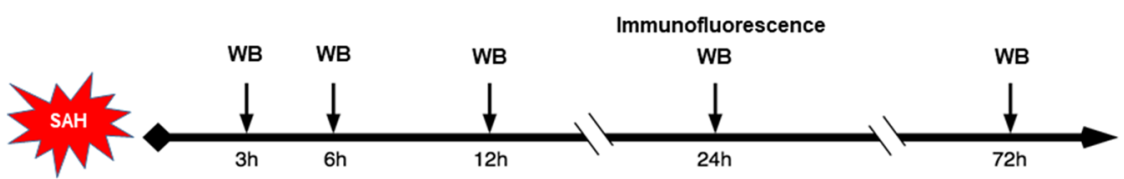

Groups: Sham, SAH-3h, SAH-6h, SAH-12h, SAH-24h, SAH-48h, SAH-72h.

Experiment 2. Apelin-13 treatment could improve short-term(24h) neurological functions.

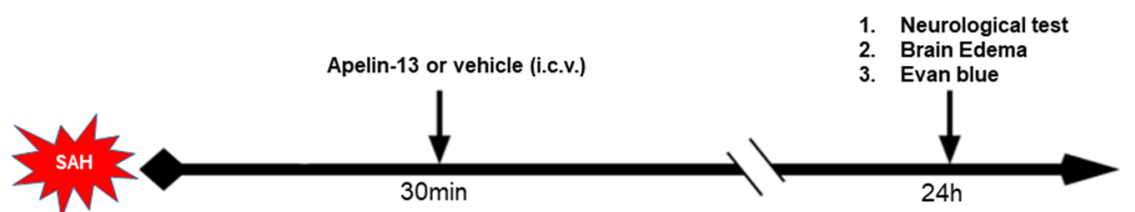

Groups: Sham, SAH + vehicle, SAH + apelin-13 (15ug/kg), SAH + apelin-13 (50ug/kg), SAH + apelin-13 (150ug/kg).

Experiment 3. Apelin-13 treatment could improve long-term(21d) neurological functions.

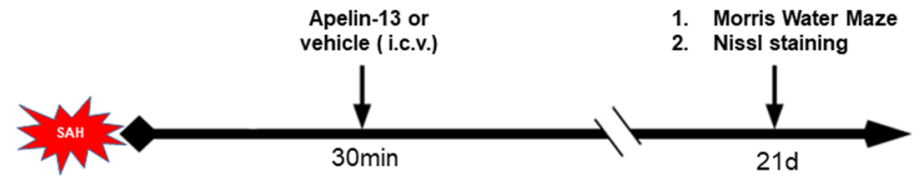

Groups: Sham, SAH + vehicle, SAH + apelin-13 (Best dosage)

Experiment 4. Knockdown of APJ with siRNA abolished the anti-inflammation effects of apelin-13.

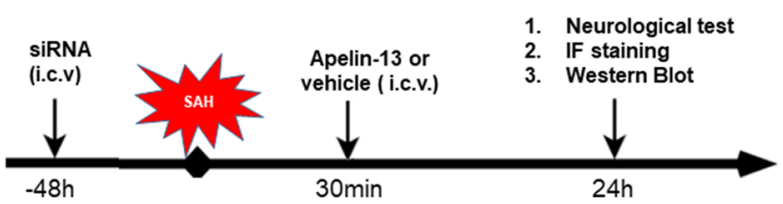

Groups: Sham, SAH + vehicle, SAH + apelin-13 (Best dosage), SAH + apelin-13 + scrRNA, SAH + apelin-13 + APJ siRNA.

Experiment 5. Dorsomorphin reversed the anti-inflammation effects of apelin-13.

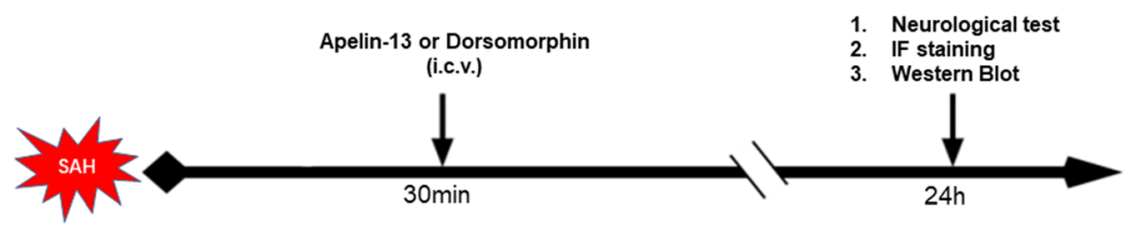

Groups: Sham, SAH + vehicle, SAH + apelin-13 (Best dosage), SAH + apelin13 + Dorsomorphin.

Fig. 1 Experimental design and animal groups 
In the fifth step, to assess the role of AMPK on the anti-neuroinflammatory effects of exogenous apelin-13, the selective AMPK inhibitor dorsomorphin $(0.1 \mu \mathrm{mol}$, in $10 \mu \mathrm{l} 20 \% \mathrm{DMSO}$ ) was administrated (i.c.v.) $30 \mathrm{~min}$ after the induction of SAH [41]. Rats were randomly distributed into sham, SAH + vehicle, SAH + apelin-13 $(50 \mu \mathrm{g} / \mathrm{kg})$, and $\mathrm{SAH}+$ apelin-13 + dorsomorphin. The ipsilateral/left cerebral cortex from each group was sampled for Western blot analysis, ROS, and immunofluorescence staining $24 \mathrm{~h}$ after $\mathrm{SAH}$.

\section{SAH model in rats}

The endovascular perforation model was utilized in this study [33]. General anesthesia was induced by peritoneal injection with pentobarbital $(40 \mathrm{mg} / \mathrm{kg})$ before the carotid artery (CA) and its bifurcation was necessarily exposed, and then a 4-0 sharpened nylon suture was inserted in the external carotid artery (ECA), which was dissected in advance. The suture marched along the internal carotid artery (ICA) to arrive at the intracranial bifurcation of the anterior cerebral artery (ACA) and middle cerebral artery (MCA), where a perforation was executed (Fig. 2a). All the perforations were performed on the left side. Therefore, the ventral side of the left hemisphere was collected for further study. Additionally, the degree of SAH was quantitatively assessed via a new grading system ([34], Additional file 1: Figure S1). The rats in the sham group received the same procedures except perforation. The rat with SAH grade less than 7 was excluded from this study.

\section{Mortality and behavior assessment}

The timing of tests was set at $24 \mathrm{~h}$ after SAH induction, depending on the time-course in which the content of apelin and APJ peaked at the point-in-time. Therefore, we calculated the mortality rate and assessed the neurological functions at $24 \mathrm{~h}$ using the previously described modified Garcia scoring system and beam balance test
A

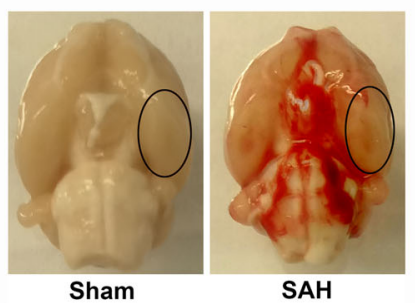

D

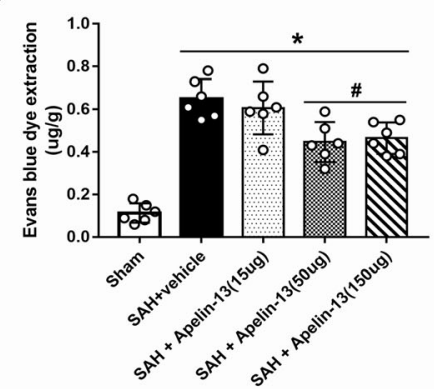

H

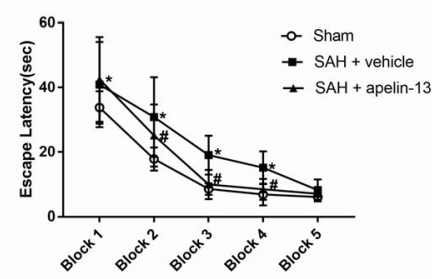

B
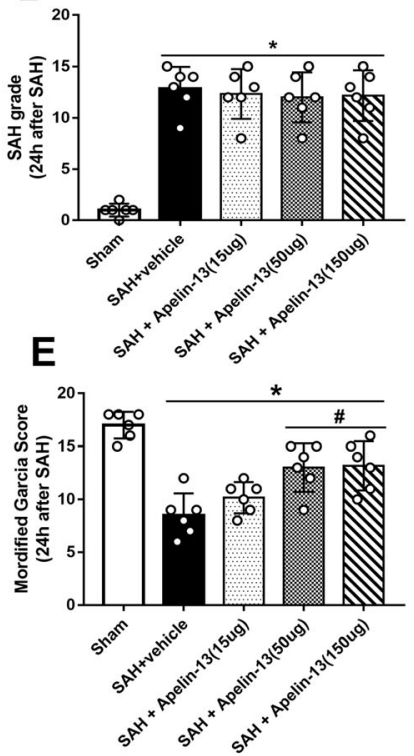

I

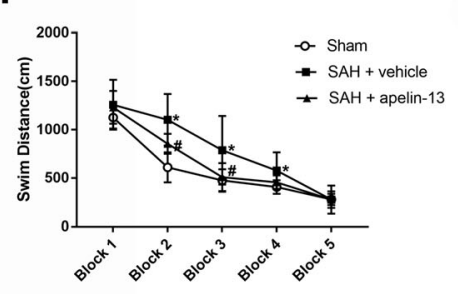

C

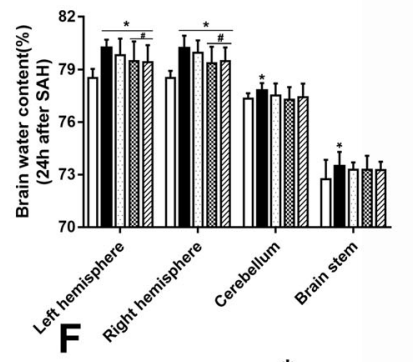

$\square$ Sham

- SAH+vehicle

$\square$ SAH + apelin-13(15ug/kg) Aㅐ + apelin-13(50ug/kg) 프 SAH + apelin-13(150ug/kg)

Fig. 2 Effects of exogenous apelin-13 on neurological functions, brain edema, and BBB leakage. a Representative pictures of brains in Sham and $\mathrm{SAH}$ groups. b SAH grade of each group. c The quantification of brain water content $24 \mathrm{~h}$ after SAH. $\mathbf{d}$ The quantification of Evans blue dye extravasation $24 \mathrm{~h}$ after SAH ( $n=6$ for each group). e, $\mathbf{f}$ Modified Garcia score and beam balance score; the effects of apelin-13 on long-term neurobehavioral outcomes after SAH. h Escape latency and i swim distance of Morris water maze on days 21 to 25 after SAH. $\mathbf{j}$ Probe quadrant duration. The bars represent the mean $\pm \mathrm{SD}$. ${ }^{*} P<0.05$ versus sham, ${ }^{*} P<0.05$ versus $\mathrm{SAH}+$ vehicle at $24 \mathrm{~h}, n=10$ per group 
[11]. The total Garcia score was graded with a scale ranging from 1 to 18. Long-term neurobehavior assessments were conducted with Morris water maze on 2125th day after SAH. We performed the water maze test according to a previous report [10,21, 47].

\section{Brain water content}

We used the wet-dry method to evaluate the brain water content $24 \mathrm{~h}$ after SAH. Briefly, the rats received euthanasia and the brains were collected quickly and then separated into four parts: left hemisphere, right hemisphere, cerebellum, and brain stem. Afterwards, each part of the brain was weighed (wet weight). Next, each part was put in an oven for $72 \mathrm{~h}\left(105^{\circ} \mathrm{C}\right.$, dry weight). We then calculated the brain water content as follows: [ (wet weight dry weight $) /($ wet weight $)] \times 100 \%[49,50]$.

\section{Evans blue staining}

Evans blue (EB) staining was applied to evaluate the blood-brain barrier integrity. Two percent EB solution $(8 \mathrm{ml} / \mathrm{kg}$, Sigma-Aldrich) was intraperitoneally injected after anesthesia. After $24 \mathrm{~h}$, the rats received transcardiac perfusion with 0.1 M PBS. Next, the brain was removed and homogenized in 50\% trichloroacetic acid. The sample was incubated in a water bath $\left(50{ }^{\circ} \mathrm{C}\right)$ for 48 $\mathrm{h}$ and centrifuged at $15,000 \times \mathrm{g}$ for $30 \mathrm{~min}$. The supernatant was detected by spectrofluorophotometry at 620 nm [51].

\section{Immunohistochemistry staining}

The rats received trans-cardiac perfusion with $0.1 \mathrm{M}$ PBS after anesthetization, followed by $4 \%$ paraformaldehyde $(\mathrm{pH}=7.4)$. We then collected the brains and put them into $4 \%$ PFA for post-fixation $\left(4^{\circ} \mathrm{C}, 24 \mathrm{~h}\right)$. Then, the brains were immersed in sucrose solution $(30 \%, 2$ days). Next, the brains were coronally sliced into $10 \mu \mathrm{m}$ sections, which were then fixed on slides and used for immunofluorescence staining, and blocked with 5\% normal donkey serum at room temperature for $2 \mathrm{~h}$ and then incubated with primary antibodies at $4{ }^{\circ} \mathrm{C}$ overnight: APJ (1:100, Santa Cruz sc-517300), IL-1 $\beta$ (1:100, Santa Cruz sc-52012), Iba-1 (1:500, Abcam ab5076), GFAP (1:500, Abcam ab7260), and NeuN (1:500, Abcam ab177487). Secondary antibodies were then applied at room temperature for $2 \mathrm{~h}$. Finally, the sections were assessed with a fluorescence microscope (Olympus, Tokyo, Japan) and images were further processed using Photoshop 13.0 (Adobe Systems Inc., Seattle, WA, USA). The number of Iba-1 and myeloperoxidase (MPO) positive cells was counted in three different fields in the ipsilateral cortex from five random coronal sections per brain using a magnification of $\times 200$ over a microscopic field of 0.01 $\mathrm{mm}^{2}$, and data were expressed as cells/field.

\section{Small interfering RNA and intracerebroventricular injection}

Intracerebroventricular injection was performed according to a previous report [47]. After the rats were anesthetized, we used a cranial drill to make a burr hole at $1 \mathrm{~mm}$ posterior to the bregma and $1.5 \mathrm{~mm}$ right lateral to the midline. A total volume of $10 \mu \mathrm{l}(500 \mathrm{pmol}$, sterile saline $)$ of rat APJ siRNA (Thermo Fisher Scientific, USA) was then injected into the right ventricle $(3.5 \mathrm{~mm}$ depth below the skull) with a pump at the rate of $0.5 \mu \mathrm{l} / \mathrm{min} 48 \mathrm{~h}$ before $\mathrm{SAH}$. Moreover, the same volume of scramble siRNA (Thermo Fisher Scientific, USA) was intracerebroventricularly injected as a negative control. The needle was kept in place for $5 \mathrm{~min}$. Finally, the burr hole was closed with bone wax and the incision was sealed with sutures. We chose this timepoint based on several papers previously reported $[25,45]$. The siRNA needs at least $24 \mathrm{~h}$ to exert its knockdown functions. Therefore, the 48th hour before $\mathrm{SAH}$ is a reasonable timepoint for giving siRNA.

\section{Western blot analysis}

The rats first received transcardiac perfusion with $0.1 \mathrm{M}$ PBS after being anesthetized. The ipsilateral/left cerebral cortex of the rat was then sampled and processed as previously being reported [23]. Briefly, we collected the left basal cortical specimen $24 \mathrm{~h}$ after SAH and further processed the samples. Protein $(40 \mu \mathrm{g})$ from each sample was added for electrophoresis $(80 \mathrm{~V}, 30 \mathrm{~min} ; 120 \mathrm{~V}, 50 \mathrm{~min})$ and then transferred to the polyvinylidene fluoride membranes at $250 \mathrm{~mA}$ for $1 \mathrm{~h}$. Then, the membrane was blocked for $1 \mathrm{~h}$ at room temperature with $5 \%$ non-fat blocking grade milk (Bio-Rad, Hercules, CA, USA). Next, the membrane was incubated with primary antibodies overnight $\left(4{ }^{\circ} \mathrm{C}\right)$ : apelin-13 (1:500, Phoenix Pharmaceuticals H-057-30), APJ (1:500, Santa Cruz sc-517300), Bip/ GRP78 (1:2000, Abcam ab21685), TXNIP (1:2000, Abcam ab188865), AMPK (1:1000, Cell signaling \#5832), p-AMPK (1:1000, Cell signaling \#2535), IL-1ß (1:2000, Santa Cruz SC-23459), TNF- $\alpha$ (1:5000, Abcam ab6671), NLRP3 (1:1000, NOVUS, CO), and $\beta$-actin (1:5000, Abcam ab8226). Secondary antibodies (1:10000, Zhongshan Gold Bridge) were then applied at room temperature for $1 \mathrm{~h}$. Finally, we used ECL Plus chemiluminescence reagent kit (Amersham Bioscience, Arlington Heights, IL) to expose and detect the protein. Then, ImageJ software (NIH) was used to measure intensity. The results were displayed as relative density (grayscale value of the target proteins/ $\beta$-actin or total proteins).

\section{Evaluation of ROS level}

We detected the level of ROS in brain tissues using a ROS assay kit (JianCheng, China). Briefly, the brain tissues were lysed in $0.01 \mathrm{~mol} / \mathrm{L}$ PBS with centrifugation at $4500 \times g$ for $10 \mathrm{~min}$. We then collected the supernatant 
$(190 \mu \mathrm{l})$ and mixed it with DCFH-DA $(10 \mu \mathrm{l}, 1 \mathrm{~mol} / \mathrm{L})$ in a micro-well at room temperature for $30 \mathrm{~min}$. Next, fluorophotometry was used to detect the sample. We measured the protein levels of different samples using a detergent-compatible protein assay kit (Bio-Rad, Hercules, CA, USA). The ROS levels were measured in the form of fluorescence/milligram protein.

\section{Statistical analysis}

Results were displayed as the mean \pm SD. SPSS 22.0 software was applied to analyze the data (IBM, USA). First, we assessed the normality of the data. If the data met the requirement of satisfied normality and homogeneity of variance, one-way analysis of variance (ANOVA) is followed by multiple comparisons between different groups using Tukey's post hoc test. For the data that failed the normality test, non-parametric statistics was applied. Additionally, two-way repeated-measures ANOVA was applied to analyze the data of long-term neurological functions and brain water content. Statistical significance was set at $P<0.05$.

\section{Results}

Physiological data

The data regarding physiology such as body temperature, heart rate, blood pressure, blood glucose, $\mathrm{PO}_{2}$, and $\mathrm{PCO}_{2}$ were monitored during the study, and no significant difference between any two groups was noted (Additional file 1: Table S2).

\section{Mortality rates and SAH grade score}

The total number of rats used in this study was 312; 53 rats were in the sham group, 18 rats were treated as
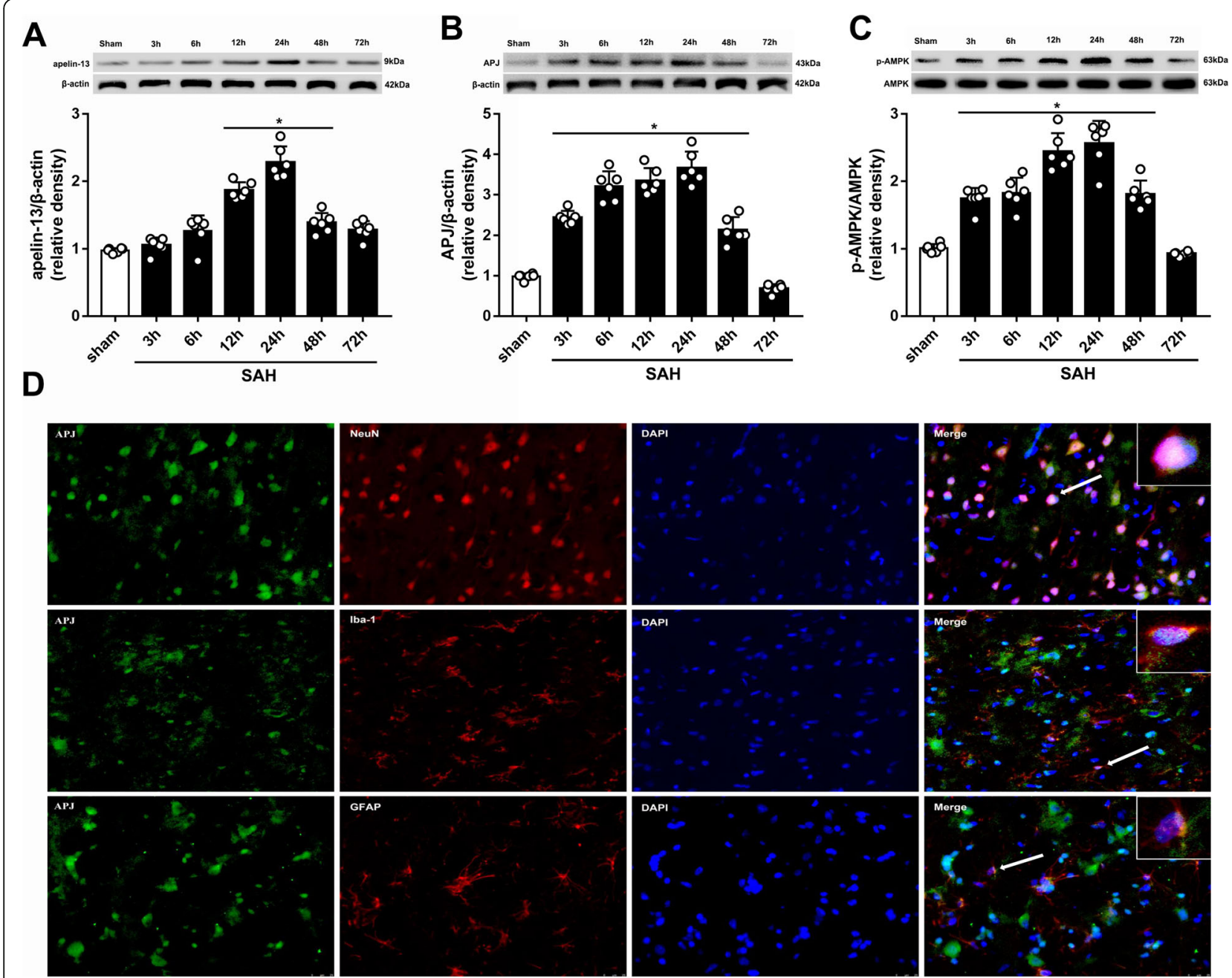

Fig. 3 Expression of apelin-13, APJ, and p-AMPK. a Representative Western blot images and quantitative analyses of apelin-13 time-course from the left hemisphere after SAH. b Representative Western blot images and quantitative analyses of APJ time course from the left hemisphere after $\mathrm{SAH}$. c Representative Western blot images and quantitative analyses of p-AMPK time course from the left hemisphere after SAH; $n=6$ for each group. The bars represent the mean \pm SD. ${ }^{*} P<0.05$ versus sham. $\mathbf{d}$ Representative microphotographs of immunofluorescence staining showing localization of APJ (green) with NeuN, iba-1, and GFAP (red) $24 \mathrm{~h}$ after SAH ( $N=2$ for each group). Scale bar $=50 \mu \mathrm{m}$ 
naïve groups, and 241 rats received perforation surgery. The overall mortality of SAH was $16.18 \%$. No rats died in the sham group, and 9 rats were excluded owing to mild SAH (SAH grade < 7) (Additional file 1: Table S1). No significant differences in SAH grade among all SAH groups were observed (Fig. 2b). Blood clots were mainly observed around the circle of Willis and ventral brain stem after SAH induction. We chose the region of basal cortex of the left hemisphere for immunochemistry staining and Western blot analysis (Fig. 2a).

\section{Brain edema, BBB permeability, and neurological functions at $24 \mathrm{~h}$ after $\mathrm{SAH}$}

Three dosages of the apelin-13 $(15 \mu \mathrm{g} / 10 \mu \mathrm{l}, 50 \mu \mathrm{g} / 10 \mu \mathrm{l}$, $150 \mu \mathrm{g} / 10 \mu \mathrm{l})$ were intracerebroventricularly administered $1 \mathrm{~h}$ after SAH. Brain water content and BBB integrity were evaluated $24 \mathrm{~h}$ after SAH. The results showed that brain water content in the $\mathrm{SAH}+$ vehicle group was significantly increased compared to the sham group $(P<$ 0.05 , Fig. 2c). The use of middle and high doses of apelin-13 significantly reduced the brain edema in the left and right hemispheres $(P<0.05$ versus $\mathrm{SAH}+$ vehicle, $n=6$, Fig. 2c), while no differences between these two groups were observed. For the cerebellum and brain stem, no significant differences were observed after the use of apelin-13 $(P>0.05$ versus $\mathrm{SAH}+$ vehicle, Additional file 1: Figure S2). In addition, the Evans blue dye significantly leaked in the $\mathrm{SAH}+$ vehicle group compared to the sham group $(P<0.05$, Fig. $2 \mathrm{~d})$. However, rats treated with middle and high doses of apelin-13 had less dye leakage compared to the rats in the $\mathrm{SAH}+\mathrm{ve}-$ hicle group $(P<0.05$, Fig. $2 d)$. The induction of SAH greatly aggravated the neurological deficits, which were significantly relieved by middle and high doses of apelin-

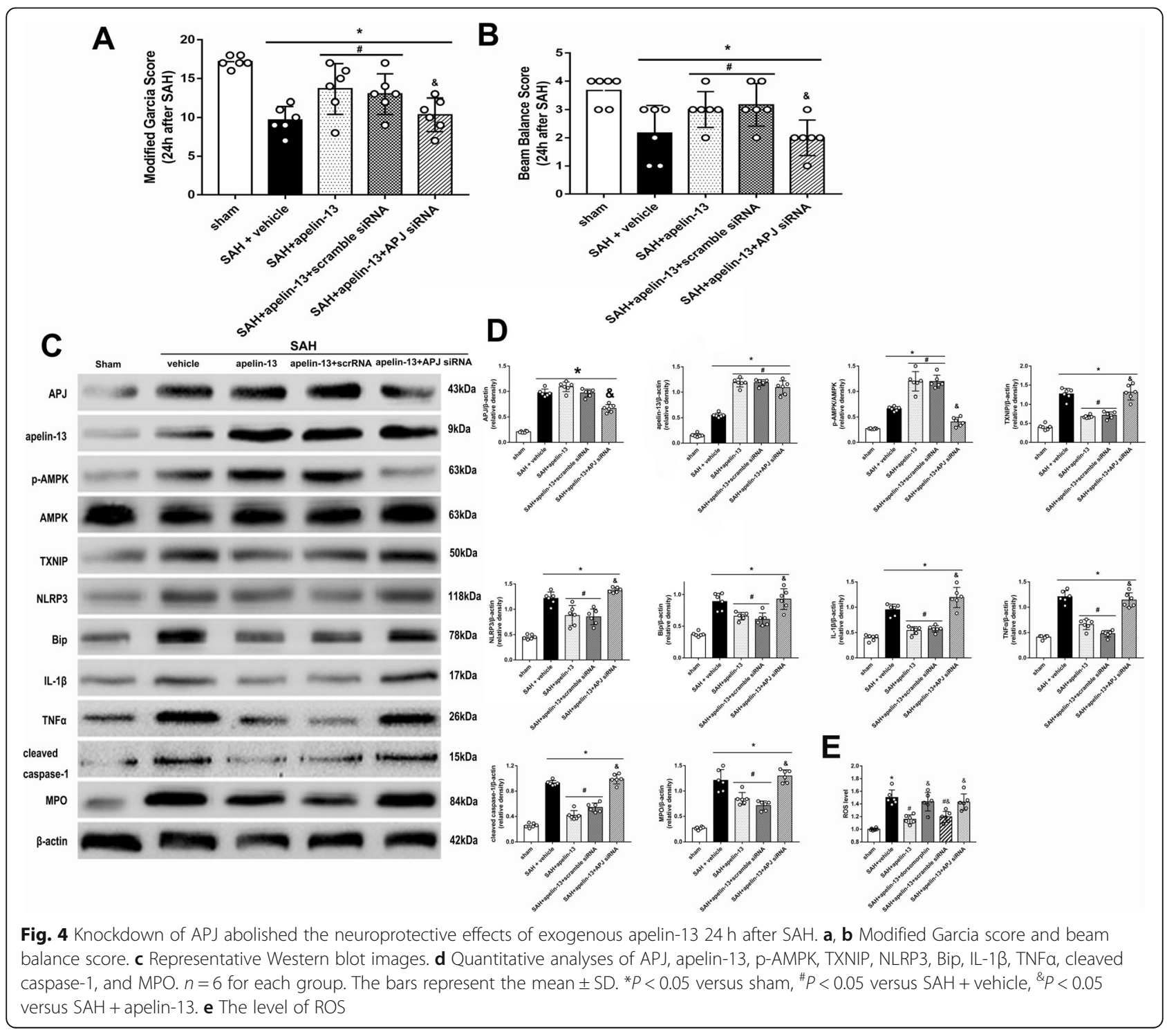


13 (Fig. 2e, f). However, there was also no significant difference between the groups receiving middle and high doses of apelin-13. Therefore, we chose the middle dose of apelin-13 for future experiments.

\section{Administration of apelin-13 improved long-term spatial learning and memory after SAH}

The escape latency and swim distance for the rats to find the platform significantly increased in the $\mathrm{SAH}+$ vehicle group compared to the sham group (Fig. $2 \mathrm{~h}$, i). After treatment with apelin-13, there was a significant decrease in escape latency on blocks 2,3 , and 4 as well as a shorter swim distance on blocks 2 and 3 compared to the SAH + vehicle group (Fig. 2h, i). Regarding the probe quadrant trial, rats in the SAH + vehicle group stayed a shorter time in the target quadrant compared to rats in the sham group. However, rats in the SAH + apelin-13 group stayed a longer time than the rats in the SAH + vehicle group (Fig. 2j).

Expression level of apelin-13, APJ, and p-AMPK after SAH The results showed that the level of apelin-13 increased at $12 \mathrm{~h}$ and peaked at $24 \mathrm{~h}$ after SAH (Fig. 3a), while the levels of APJ and p-AMPK started to increase at $6 \mathrm{~h}$ and peaked at $24 \mathrm{~h}$ after SAH (Fig. 3b, c).

\section{Cellular location of APJ after SAH}

The results of double immunofluorescence staining suggested that APJ was expressed on all types of cells. The percentage that APJ could be colocalized with the neuron or microglia is much higher than that of astrocytes in the cortex at $24 \mathrm{~h}$ after SAH (Fig. 3d).

\section{Anti-oxidative stress and anti-inflammation effects of exogenous apelin-13 is partly abolished by APJ siRNA at $24 \mathrm{~h}$ after $\mathrm{SAH}$}

The administration of exogenous apelin-13 significantly improved the neurological functions after SAH $(P<0.05$, Fig. 4a, b). In addition, exogenous apelin-13 further increased the level of p-AMPK, which suppressed the expression of downstream targets, including TXNIP, NLRP3, Bip, cleaved caspase-1, IL- $1 \beta$, and TNF $\alpha$, and also reduced the MPO levels $(P<0.05$, Fig. $4 \mathrm{c}, \mathrm{d})$ compared to the $\mathrm{SAH}+$ vehicle group. However, the knockdown of

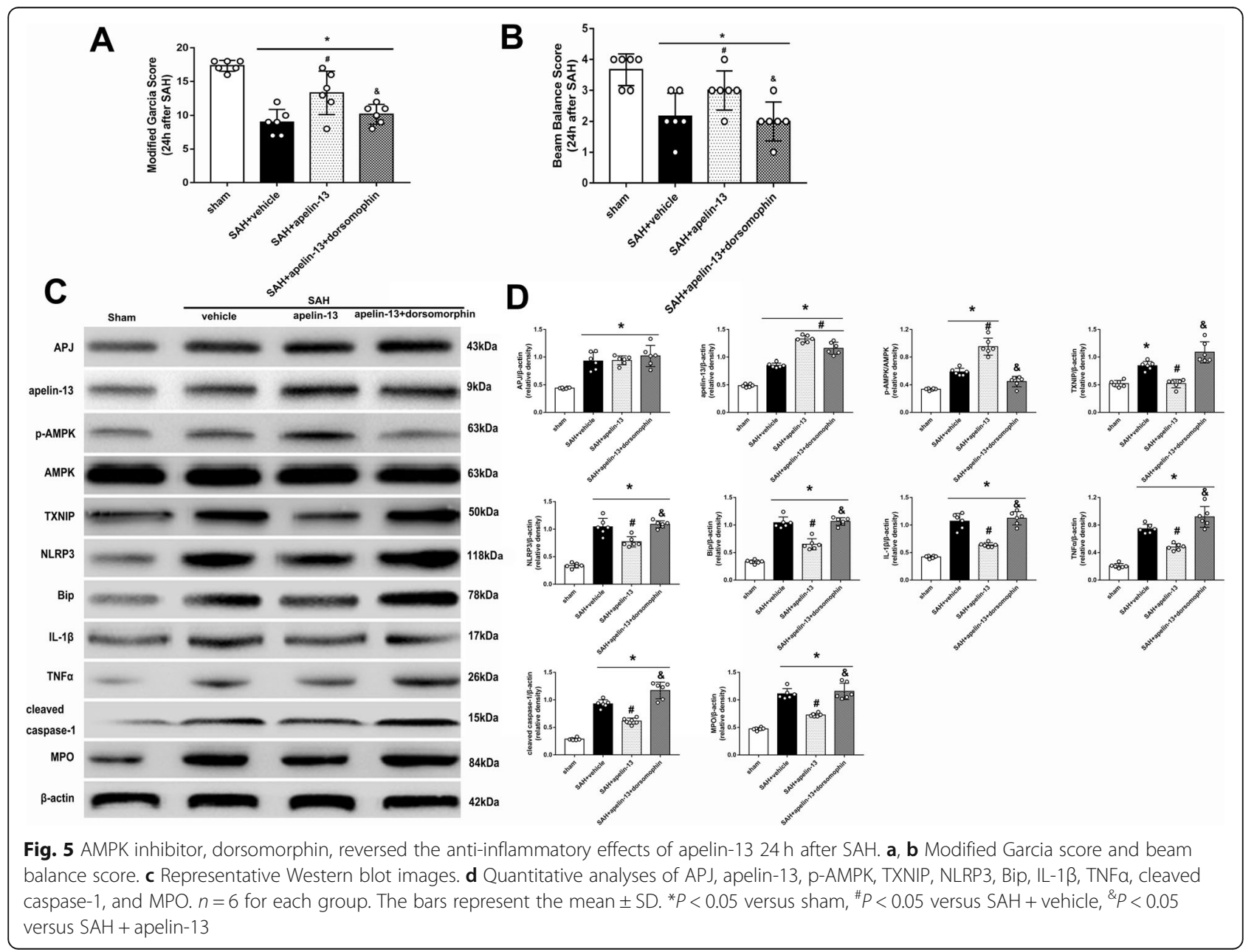


APJ with APJ siRNA partly offset anti-oxidative stress and anti-inflammation effects of exogenous apelin-13. The results showed that the knockdown of APJ inhibited the expression of APJ and pAMPK $24 \mathrm{~h}$ after $\mathrm{SAH}$ and increased the levels of TXNIP, NLRP3, Bip, cleaved caspase-1, IL-1 $\beta$, TNF $\alpha$, MPO, and ROS $(P<0.05$, Fig. 4c-e). Moreover, exogenous apelin-13 decreased the level of ROS, which is also partly abolished by APJ siRNA $(P<0.05$, Fig. 4e $)$.

AMPK inhibitor, dorsomorphin, reversed the antiinflammatory effects of apelin-13 $24 \mathrm{~h}$ after SAH

The AMPK inhibitor, dorsomorphin, was used to further verify the neuroprotective pathway of apelin-13 after SAH. Dorsomorphin treatment significantly aggravated the neurological deficits $(P<0.05$, Fig. 5a, b). Furthermore, the administration of dorsomorphin reduced the level of p-AMPK and increased the levels of TXNIP, NLRP3, Bip, cleaved caspase-1, IL-1 $\beta$, TNF $\alpha$, MPO, and ROS $(P<0.05$, Figs. $4 \mathrm{e}$ and $5 \mathrm{c}, \mathrm{d})$. MPO positive and iba-1 positive cells were also significantly increased after the use of dorsomorphin $(P<0.05$, Figs. $6 a$, $b$ and $7 \mathrm{a}, \mathrm{b})$. Besides, we did an additional experiment where the dorsomorphin is given alone to see the effects of dorsomorphin. The results showed that the inhibition of AMPK significantly increased the inflammatory molecules (IL$1 \beta$, TNF $\alpha$, MPO, etc.; $P<0.05$, Fig. 8a, b).

\section{Assessment of the depletion efficiency of APJ siRNA with naïve rats}

In order to test the depletion efficiency of APJ siRNA, we intraventricularly injected APJ siRNA in naïve rats. The results showed that APJ siRNA reduced the level of APJ by $46 \%$ on average (Additional file 1: Figure S3).

\section{Discussion}

In this study, we explored the neuroprotective effects of apelin-13 through a new mechanism mediated by the APJ/AMPK/TXNIP/NLRP3 pathway in experimental $\mathrm{SAH}$ in rats. The novel findings of this study include the following: (1) endogenous apelin-13, APJ, and p-AMPK levels were significantly increased and peaked in the brain $24 \mathrm{~h}$ after SAH; APJ was mainly expressed in the

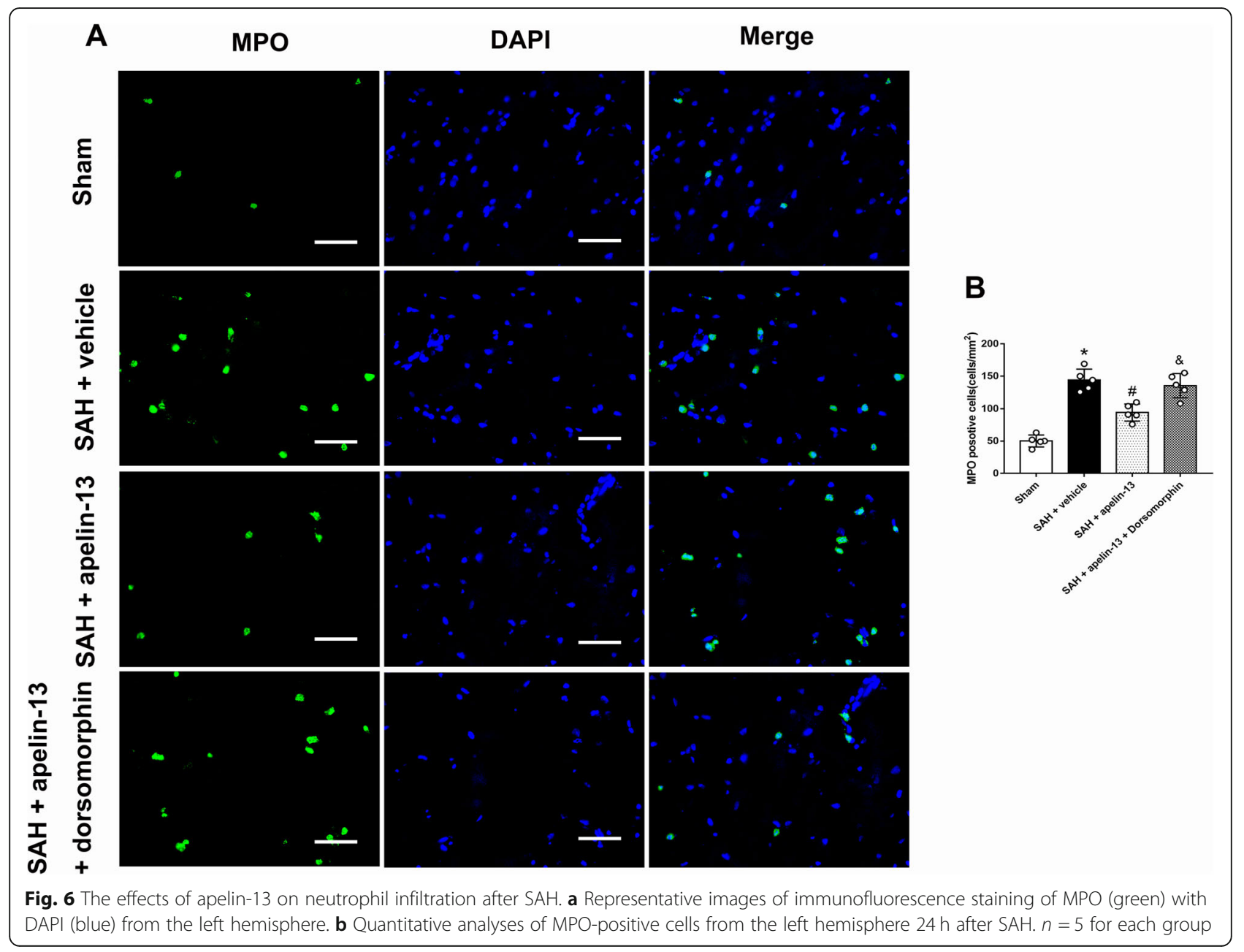




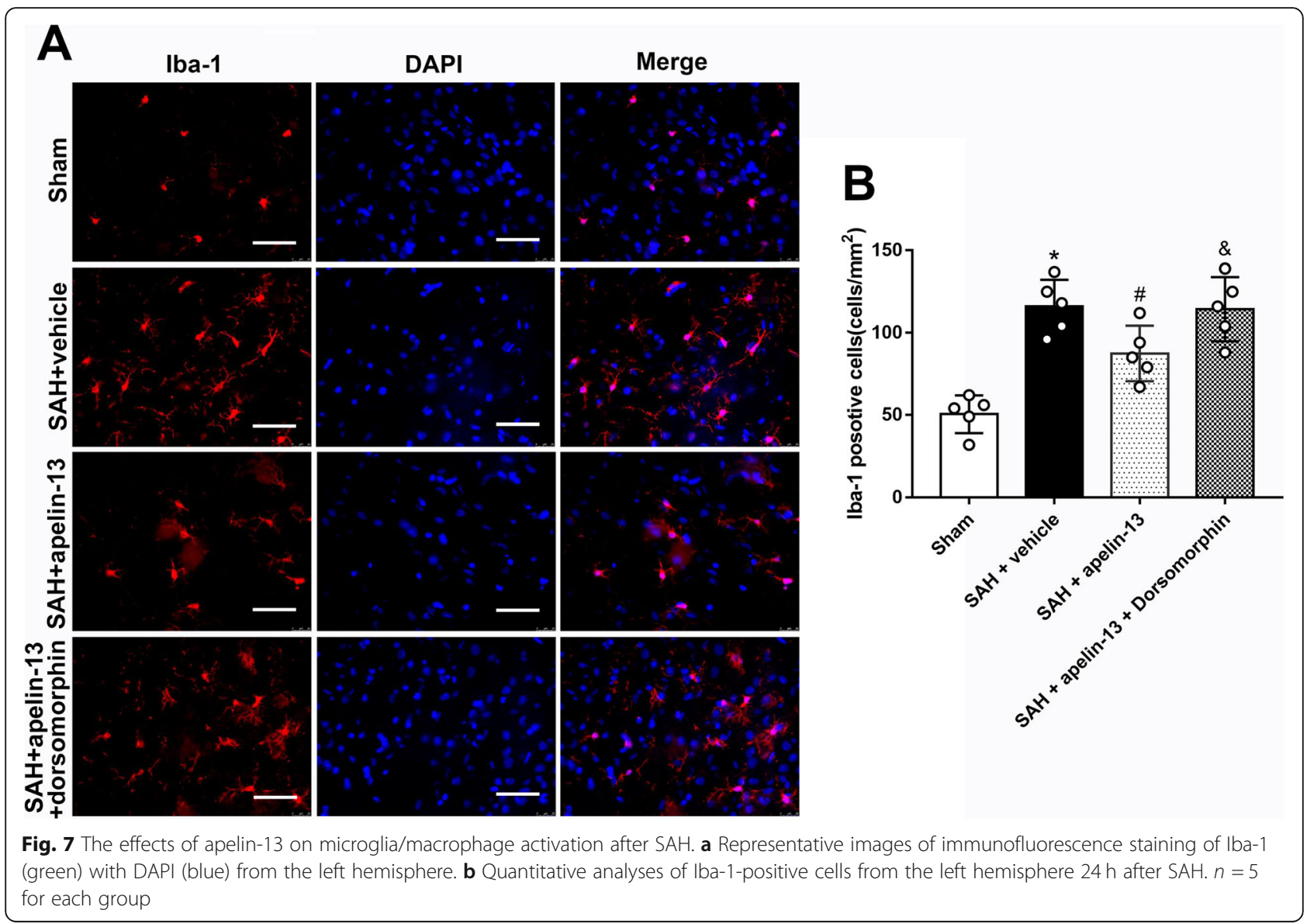

microglia and neurons; (2) administration of exogenous apelin-13 significantly alleviated neurological functions, attenuated brain edema, and preserved BBB integrity; (3) treatment with apelin-13 significantly improved longterm spatial learning and memory after SAH; (4) exogenous apelin-13 suppressed microglia activation, prevented ER stress from overactivation, and reduced the levels of TXNIP, NLRP3, Bip, cleaved caspase-1, IL-1 $\beta$, TNF $\alpha$, MPO, and ROS; (5) siRNA knockdown of APJ also abolished the neuroprotective effects of apelin-13 on neuroinflammation and oxidative stress; and (6) the AMPK inhibitor dorsomorphin also reversed the neuroprotective effects of apelin-13 $24 \mathrm{~h}$ after SAH. Taken together, the results show that exogenous apelin-13 binding to APJ can attenuate early brain injury by reducing ER stress-mediated oxidative stress and neuroinflammation, which is at least partly mediated by the AMPK/TXNIP/NLRP3 signaling pathway.

Increasing studies show the important roles of neuroinflammation and ER stress-mediated oxidative stress in early brain injury after SAH [7, 42]. ER is an organelle that mainly manipulates protein synthesis and further processing. Insults that perturb ER function leads to ER stress [27], which could be induced by inflammation, oxidative stress, or mitochondrial calcium overloading [18]. After induction of SAH, blood immediately spreads through the subarachnoid space and then covers the cerebral cortex with a thick blood clot [22]. The resulting accumulations of cell breakdown components, dysfunctional organelles, inflammatory factors, ROS, and other cytokines can inhibit normal protein folding, which activates UPR/ER stress and finally causes irreversible neurological deficits [42]. In turn, the overactivation of ER stress aggravates the inflammatory response and oxidative stress, including further microglia activation and leukocyte infiltration into the brain, which subsequently trap in a vicious circle to exacerbate brain injury after stroke [13, 39]. Therefore, suppressing microglia activation and neutrophil infiltration and reducing oxidative stress are beneficial to ameliorate early brain injury after SAH.

Apelin-13 has been reported to provide neuroprotection against cerebral ischemic stroke via antineuroinflammation, anti-oxidative stress, or preventing ER stress from overactivation [3, 4, 43]. Leeper et al. also found that apelin-13 significantly reduced aneurysm formation by decreasing proinflammatory cytokine and chemokine activation [20]. Zhang et al. reported that 


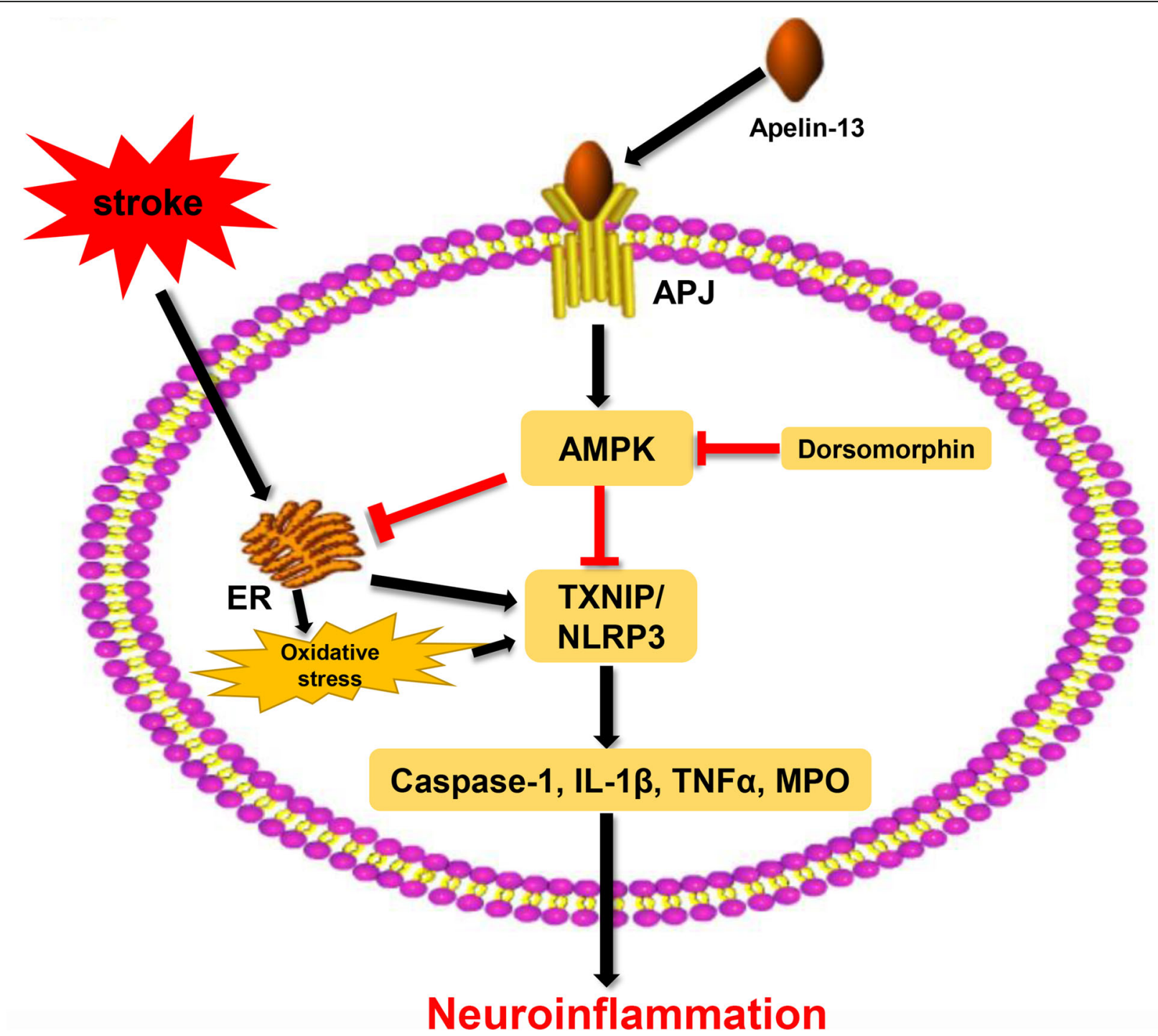

Fig. 8 The effects of dorsomorphin on the rats with SAH. a Representative Western blot images. $\mathbf{b}$ Quantitative analyses of $p-A M P K$, IL-1 $\beta$, TNFa, cleaved caspase-1, MPO. $n=6$ for each group. The bars represent the mean \pm SD. ${ }^{*} P<0.05$ versus sham, ${ }^{\#} P<0.05$ versus SAH + vehicle

apelin-13 could attenuate cisplatin-induced cardiotoxicity by reducing ROS-mediated DNA damage $[49,50]$. In the present study, we found that the administration of apelin-13 significantly improved neurological functions and reduced the inflammatory response, neutrophil infiltration, and oxidative stress including the levels of Bip, IL-1 $\beta$, TNF $\alpha$, MPO, and ROS. These observations suggest that apelin-13 could suppress neuroinflammation and oxidative stress by inhibiting the expression of inflammatory molecules and preventing overactivation of the ER stress response following SAH.

Apelin-13 exerts its functions through binding to its receptor APJ. Apelin-13 was reported to protect against multiple organ injury following hemorrhagic shock by binding to APJ and decreasing the number of inflammatory cells and swollen mitochondria in cells [29]. In the model of ischemic stroke, the apelin-13/APJ system suppressed the activation of microglia and astrocyte and reduced neutrophil infiltration [40]. In the current study, the results of double immunofluorescence staining showed that APJ was expressed in microglia and neurons. Moreover, the knockdown of APJ with specific siRNA significantly abolished the anti-inflammation and anti-oxidative stress property of exogenous apelin-13, which could suppress microglia activation and diminish pro-inflammatory TNF- $\alpha$ and IL- $1 \beta$ levels as well as the levels of ROS and MPO. Therefore, it is reasonable to speculate that APJ mediates apelin-13-induced antiinflammation and anti-oxidative effects after SAH.

AMPK has been reported to be the key factor in regulating the inflammatory response and ER stress $[16,17$, 19, 37]. An et al. reported the activation of AMPK 
increased neuronal apoptosis and brain injury after SAH [2]; however, more studies hold the opinion that phosphorylation of AMPK provides neuroprotection in acute stroke attack as follows. The level of AMPK was increased after stroke, which could further suppress the activation of microglia and reduce the infiltration of neutrophil into brain tissues [38]. Increased AMPK reinforced Nrf2-mediated antioxidant pathways [1]. Ying et al. found that AMPK could attenuate glutamate neurotoxicity in the hippocampus by suppressing the ER stress-mediated TXNIP/NLRP3 inflammasome [16]. Moreover, enhanced AMPK phosphorylation reduced ROS and ER stress-mediated TXNIP/NLRP3 inflammasome [52]. Recently, increasing evidence has indicated the importance of the NLRP3 inflammasome in the post-SAH inflammatory response $[9,28]$. Once activated, the NLRP3 inflammasome causes transformation of procaspase- 1 into cleaved caspase- 1 and maturation of IL-1 $\beta$ and IL-18, which subsequently contributes to inflammation after SAH [9]. In addition, some studies showed that AMPK is a downstream target of apelin-13mediated anti-inflammation and anti-oxidative stress during ischemic injury in the brain and heart [35, 43]. In addition, Apelin-13 attenuated the systemic inflammatory response and promoted survival after severe burn by inhibiting the activation of the NLRP3 inflammasome in rats [5]. In this study, we observed that the levels of APJ and AMPK increased after SAH. However, the extent of endogenous APJ and AMPK upregulation was not enough to reduce the level of the NLRP3 inflammasome and inflammatory cytokines. After the use of exogenous apelin-13, the level of AMPK was further increased, which significantly inhibited the activation of the NLRP3 inflammasome and reduced the levels of Bip, cleaved caspase- 1 , IL-1 $\beta$, TNF- $\alpha$, MPO, and ROS. However, the AMPK inhibitor reversed the antiinflammatory and anti-oxidative stress effects and upregulated the expression of NLRP3, cleaved caspase-1, IL$1 \beta$, TNF $\alpha, \mathrm{MPO}$, and ROS, thereby worsening the neurological deficits. Therefore, our findings supported the hypothesis that the anti-inflammatory and antioxidative stress effects of exogenous apelin-13 is mediated at least in part through the AMPK/TXNIP/NLRP3 signaling pathway after SAH (Fig. 9).

Although this study verified the value of apelin-13 in a novel mechanism mediated via suppression of the AMPK/ TXNIP/NLRP3 signaling pathway, some limitations could not be ignored. First, the apelin-13/APJ system can display

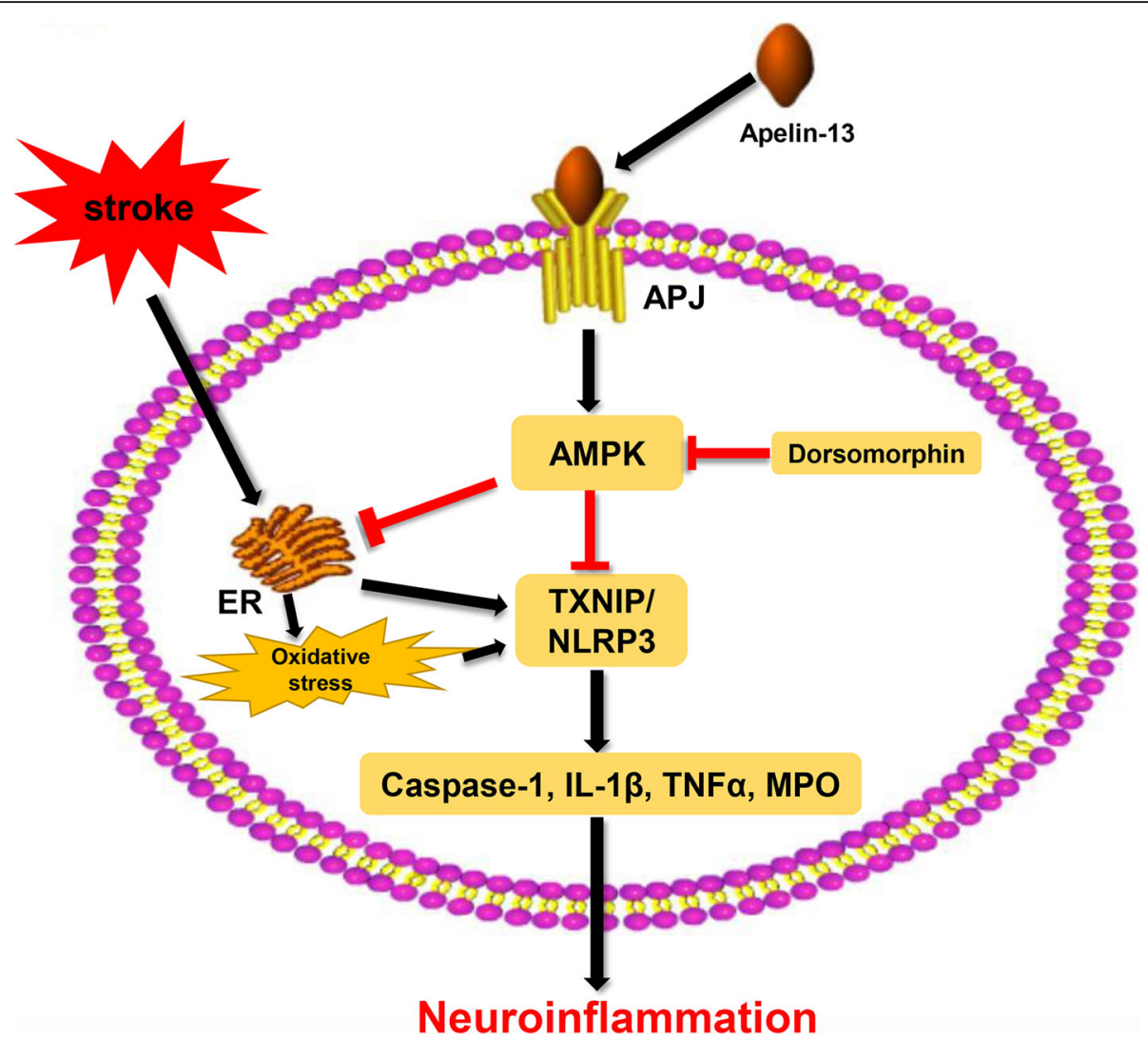

Fig. 9 The potential molecular mechanisms of apelin-13/APJ system-mediated anti-inflammatory and anti-oxidative effects via suppression of ER stress-associated AMPK/TXNIP/NLRP3 inflammasome activation 
its neuroprotective effects following SAH in many ways; however, only the AMPK-dependent pathway was studied. Second, only the anti-inflammatory and anti-oxidative characteristics of the apelin-13/APJ system were evaluated in this study, without further investigation of its roles in apoptosis or autophagy. Therefore, further studies focusing on other roles of the apelin-13/APJ system in SAH and more characteristics of the apelin-13/APJ system need to be performed.

\section{Conclusions}

Our study showed that exogenous apelin-13 binding to APJ improves neurological functions and attenuates early brain injury after SAH by reducing ER stress-mediated oxidative stress and neuroinflammation, which is at least partly mediated by the AMPK/TXNIP/NLRP3 signaling pathway. Therefore, apelin-13/APJ system can be a promising therapeutic target in the treatment of SAH.

\section{Supplementary information}

Supplementary information accompanies this paper at https://doi.org/10. 1186/s12974-019-1620-3.

Additional file 1: Table S1 Study design and animal usage. Table S2.

Rats physical data after surgeries. Figure $\mathbf{S 1}$. The ventral side was divided into six parts. SAH severity score: a grade from 0-3 is dependent on the amount of blood clot in each segment as follows: grade 0:no blood clot: 1: minimal blood clot; 2: moderate blood clot with recognizable arteries; 3: blood clot obliterating all arteries. The SAH grade was the total scores of the six parts, with minimal score of 0 and maximal score of 18. Figure S2. Effects of apelin-13 on brain edema: the quantification of brain water content of cerebellum and brain stem at $24 \mathrm{~h}$ after SAH. Figure S3. Depletion Efficiency of APJ siRNA with Naive Rats. (A) Representative Western blot images. (B) Quantitative analyses of APJ. $n=6$ for each group. The bars represent the mean $\pm S D$. ${ }^{*} p<0.05$ versus naïve.

\section{Abbreviations}

SAH: Subarachnoid hemorrhage; APJ: Apelin receptor; TXNIP: Thioredoxininteracting protein; NLRP3: NOD-like receptor pyrin domain-containing 3 protein; AMPK: Adenosine monophosphate-activated protein kinase; ER: Endoplasmic reticulum; BBB: Blood-brain barrier; MPO: Myeloperoxidase; ROS: Reactive oxygen species; EBI: Early brain injury; CA: Carotid artery; ECA: External carotid artery; ICA: Internal carotid artery; ACA: Anterior cerebral artery; MCA: Middle cerebral artery; EB: Evans blue

\section{Acknowledgements}

Not applicable.

\section{Authors' contributions}

This study was designed by WX and TL. The experiments were completed by $W X, L G$, and JZ. WX and LG performed statistical analysis. $W X$, $T L$, and AS finished writing the manuscript. JZ and AS participated in discussion development and provided expert guidance. All authors read and approved the final manuscript.

\section{Funding}

This work was funded by China Postdoctoral Science Foundation (2017 M612010), National Natural Science Foundation of China (81701144), National Natural Science Foundation of China (81371433), National Key Research and Development Program of China (2017YFC1308500), and Key Program of Science and Technology Development of Zhejiang (2017C03021).

\section{Availability of data and materials}

The datasets analyzed during the current study are available from the corresponding author on reasonable request.

\section{Ethics approval}

All animal experiments were performed according to the Institutional Animal Care and Use Committee of Zhejiang University. The procedures were conducted according to the National Institutes of Health's Guide for the Care and the Use of Laboratory Animals and the ARRIVE (Animal Research: Reporting In Vivo Experiments) guidelines.

\section{Consent for publication}

Not applicable.

\section{Competing interests}

The authors declare that they have no competing interests.

\section{Author details}

'Department of Neurosurgery, Second Affiliated Hospital, School of Medicine, Zhejiang University, 88 Jiefang Rd, Hangzhou 310009, Zhejiang, China. ${ }^{2}$ Department of Neurosurgery, Affiliated Tumor Hospital of Guangxi Medical University, Nanning, Guangxi Zhuang Autonomous Region, China. ${ }^{3}$ Brain Research Institute, Zhejiang University, Hangzhou, Zhejiang, China. ${ }^{4}$ Collaborative Innovation Center for Brain Science, Zhejiang University, Hangzhou, Zhejiang, China.

Received: 28 March 2019 Accepted: 22 October 2019

Published online: 02 December 2019

\section{References}

1. Ashabi G, Khalaj L, Khodagholi F, Goudarzvand M, Sarkaki A. Pre-treatment with metformin activates Nrf2 antioxidant pathways and inhibits inflammatory responses through induction of AMPK after transient global cerebral ischemia. Metab Brain Dis. 2015;30(3):747-54.

2. An JY, Zhou LL, Sun P, Pang HG, Li DD, Li Y, Zhang M, Song JN. Role of the AMPK signaling pathway in early brain injury after subarachnoid hemorrhage in rats. Acta Neurochir. 2015;157(5):781-92.

3. Chu H, Yang X, Huang C, Gao Z, Tang Y, Dong Q. Apelin-13 protects against ischemic blood-brain barrier damage through the effects of aquaporin-4. Cerebrovasc Dis. 2017;44(1-2):10-25.

4. Chen D, Lee J, Gu X, Wei L, Yu SP. Intranasal delivery of apelin-13 is neuroprotective and promotes angiogenesis after ischemic stroke in mice. ASN Neuro. 2015;7(5).

5. Chi Y, Chai J, Xu C, Luo H, Zhang Q. Apelin inhibits the activation of the nucleotide-binding domain and the leucine-rich, repeat-containing family, pyrin-containing 3 (NLRP3) inflammasome and ameliorates insulin resistance in severely burned rats. Surgery. 2015;157(6):1142-52.

6. Chen S, Feng H, Sherchan P, Klebe D, Zhao G, Sun X, et al. Controversies and evolving new mechanisms in subarachnoid hemorrhage. Prog Neurobiol. 2014;115:64-91.

7. de Oliveira Manoel AL, Macdonald RL. Neuroinflammation as a target for intervention in subarachnoid hemorrhage. Front Neurol. 2018;9:292.

8. Dou Y, Shen H, Feng D, Li H, Tian X, Zhang J, Wang Z, Chen G. Tumor necrosis factor receptor-associated factor 6 participates in early brain injury after subarachnoid hemorrhage in rats through inhibiting autophagy and promoting oxidative stress. J Neurochem. 2017;142(3):478-92.

9. Dong Y, Fan C, Hu W, Jiang S, Ma Z, Yan X, et al. Melatonin attenuated early brain injury induced by subarachnoid hemorrhage via regulating NLRP3 inflammasome and apoptosis signaling. J Pineal Res. 2016;60:253-62.

10. Edwards SR, Hamlin AS, Marks N, Coulson EJ, Smith MT. Comparative studies using the Morris water maze to assess spatial memory deficits in two transgenic mouse models of Alzheimer's disease. Clin Exp Pharmacol Physiol. 2014:41(10):798-806.

11. Garcia J, et al. Neurological deficit and extent of neuronal necrosis attributable to middle cerebral artery occlusion in rats. Stat Validation Stroke. 1995;26(4):627-34.

12. Huang J, Kang $S$, Park SJ, Im DS. Apelin protects against liver $X$ receptormediated steatosis through AMPK and PPARa in human and mouse hepatocytes. Cell Signal. 2017;39:84-94.

13. Keep RF, Hua $Y, X_{i} G$. Intracerebral haemorrhage: mechanisms of injury and therapeutic targets. Lancet Neurol. 2012;11:720-31. 
14. Tatemoto K, Hosoya M, Habata Y, Fujii R, Kakegawa T, Zou MX, Kawamata Y, Fukusumi S, Hinuma S, Kitada C, Kurokawa T, Onda H, Fujino M. Isolation and characterization of a novel endogenous peptide ligand for the human APJ receptor. Biochem Biophys Res Commun. 1998;251(2):471-6.

15. Li H, Yu JS, Zhang HS, Yang YQ, Huang LT, Zhang DD, Hang CH. Increased expression of caspase-12 after experimental subarachnoid hemorrhage. Neurochem Res. 2016;41(12):3407-16.

16. Li Y, Li J, Li S, Li Y, Wang X, Liu B, Fu Q, Ma S. Curcumin attenuates glutamate neurotoxicity in the hippocampus by suppression of ER stressassociated TXNIP/NLRP3 inflammasome activation in a manner dependent on AMPK. Toxicol Appl Pharmacol. 2015a;286(1):53-63.

17. Li Y, Yang J, Chen MH, Wang Q, Qin MJ, Zhang T, Chen XQ, Liu BL, Wen XD. llexgenin a inhibits endoplasmic reticulum stress and ameliorates endothelial dysfunction via suppression of TXNIP/NLRP3 inflammasome activation in an AMPK dependent manner. Pharmacol Res. 2015b;99:101-15.

18. Liu L, Liu C, Lu Y, Liu L, Jiang Y. ER stress related factor ATF6 and caspase-12 trigger apoptosis in neonatal hypoxic-ischemic encephalopathy. Int J Clin Exp Pathol. 2015;8(6):6960-6.

19. Li J, Wang Y, Wang Y, Wen X, Ma XN, Chen W, Huang F, Kou J, Qi LW, Liu B, Liu K. Pharmacological activation of AMPK prevents Drp1-mediated mitochondrial fission and alleviates endoplasmic reticulum stress-associated endothelial dysfunction. J Mol Cell Cardiol. 2015c;86:62-74.

20. Leeper NJ, Tedesco MM, Kojima Y, Schultz GM, Kundu RK, Ashley EA, Tsao PS, Dalman RL, Quertermous T. Apelin prevents aortic aneurysm formation by inhibiting macrophage inflammation. Am J Physiol Heart Circ Physiol. 2009;296(5):H1329-35.

21. Mo J, Enkhjargal B, Travis ZD, et al. AVE 0991 attenuates oxidative stress and neuronal apoptosis via Mas/PKA/CREB/UCP-2 pathway after subarachnoid hemorrhage in rats. Redox Biol. 2019;20:75-86

22. Matz PG, Copin JC, Chan PH. Cell death after exposure to subarachnoid hemolysate correlates inversely with expression of CuZn-superoxide dismutase. Stroke. 2000;31(10):2450-9.

23. Nakka VP, Gusain A, Raghubir R. Endoplasmic reticulum stress plays critical role in brain damage after cerebral ischemia/reperfusion in rats. Neurotox Res. 2010;17(2):189-202.

24. O'Dowd BF, Heiber M, Chan A, Heng HH, Tsui LC, Kennedy JL, et al. A human gene that shows identity with the gene encoding the angiotensin receptor islocated on chromosome 11. Gene. 1993;136:355-60.

25. Peng J, Pang J, Huang L, et al. LRP1 activation attenuates white matter injury by modulating microglial polarization through Shc1/PI3K/Akt pathway after subarachnoid hemorrhage in rats. Redox Biol. 2019;21:101121.

26. Pouresmaeili-Babaki E, Esmaeili-Mahani S, Abbasnejad M, Ravan H. Protective effect of neuropeptide apelin-13 on 6-hydroxydopamineinduced neurotoxicity in SH-SY5Y dopaminergic cells: involvement of its antioxidant and antiapoptotic properties. Rejuvenation Res. 2018;21(2): 162-7.

27. Roussel BD, et al. Endoplasmic reticulum dysfunction in neurological disease. Lancet Neurol. 2013;12(1):105-18.

28. Shao A, Wu H, Hong Y, Tu S, Sun X, Wu Q, et al. Hydrogen-rich saline attenuated subarachnoid hemorrhage-induced early brain injury in rats by suppressing inflammatory response: possible involvement of NF-kBpathway and NLRP3 inflammasome. Mol Neurobiol. 2016;53:3462-76.

29. Soliman M, Arafah M. Apelin protect against multiple organ injury following hemorrhagic shock and decrease the inflammatory response. Int J Appl Basic Med Res. 2015;5(3):195-9.

30. Sandal S, Tekin S, Seker FB, Beytur A, Vardi N, Colak C, Tapan T, Yildiz S, Yilmaz B. The effects of intracerebroventricular infusion of apelin-13 on reproductive function in male rats. Neurosci Lett. 2015;602:133-8.

31. Sehba FA, Hou J, Pluta RM, Zhang JH. The importance of early brain injury after subarachnoid hemorrhage. Prog Neurobiol. 2012;97:14-37.

32. Pitkin SL, Maguire JJ, Bonner TI, Davenport AP. International Union of Basic and Clinical Pharmacology. LXXIV. Apelin receptor nomenclature, distribution, pharmacology, and function. Pharmacol Rev. 2010;62(3):331-42.

33. Suzuki $\mathrm{H}$, et al. Mechanisms of osteopontin-induced stabilization of bloodbrain barrier disruption after subarachnoid hemorrhage in rats. Stroke. 2010; 41(8):1783-90

34. Sugawara T, et al. A new grading system evaluating bleeding scale in filament perforation subarachnoid hemorrhage rat model. J Neurosci Methods. 2008;167(2):327-34.

35. Tao J, Zhu W, Li Y, Xin P, Li J, Liu M, Li J, Redington AN, Wei M. Apelin-13 protects the heart against ischemia-reperfusion injury through inhibition of
ER-dependent apoptotic pathways in a time-dependent fashion. Am J Physiol Heart Circ Physiol. 2011;301(4):H1471-86

36. Wu F, Qiu J, Fan Y, Zhang Q, Cheng B, Wu Y, Bai B. Apelin-13 attenuates ER stress-mediated neuronal apoptosis by activating Gai/Gaq-CK2 signaling in ischemic stroke. Exp Neurol. 2018;302:136-44.

37. Wang Y, Ruan W, Mi J, Xu J, Wang H, Cao Z, Saavedra JM, Zhang L, Lin H, Pang T. Balasubramide derivative $3 \mathrm{C}$ modulates microglia activation via CaMKKß-dependent AMPK/PGC-1a pathway in neuroinflammatory conditions. Brain Behav Immun. 2018a;67:101-17.

38. Wang $Y$, Huang $Y, X u Y$, Ruan $W$, Wang $H$, Zhang $Y$, Saavedra JM, Zhang $L$, Huang Z, Pang T. A dual AMPK/Nrf2 activator reduces brain inflammation after stroke by enhancing microglia M2 polarization. Antioxid Redox Signal. 2018b;28(2):141-63.

39. Xie Y, Guo H, Wang L, Xu L, Zhang X, Yu L, Liu Q, Li Y, Zhao N, Zhao N, Ye $R$, Liu X. Human albumin attenuates excessive innate immunity via inhibition of microglial Mincle/Syk signaling in subarachnoid hemorrhage. Brain Behav Immun. 2017;60:346-60.

40. Xin Q, Cheng B, Pan Y, Liu H, Yang C, Chen J, Bai B. Neuroprotective effects of apelin-13 on experimental ischemic stroke through suppression of inflammation. Peptides. 2015;63:55-62.

41. Yu J, Li X, Matei N, McBride D, Tang J, Yan M, Zhang JH. Ezetimibe, a NPC1L1 inhibitor, attenuates neuronal apoptosis through AMPK dependent autophagy activation after MCAO in rats. Exp Neurol. 2018;307:12-23.

42. Yan F, Cao S, Li J, Dixon B, Yu X, Chen J, Gu C, Lin W, Chen G. Pharmacological inhibition of PERK attenuates early brain injury after subarachnoid hemorrhage in rats through the activation of Akt. Mol Neurobiol. 2017;54(3):1808-17.

43. Yang Y, Zhang XJ, Li LT, Cui HY, Zhang C, Zhu CH, Miao JY. Apelin-13 protects against apoptosis by activating AMP-activated protein kinase pathway in ischemia stroke. Peptides. 2016;75:96-100.

44. Yan F, Li J, Chen J, Hu Q, Gu C, Lin W, Chen G. Endoplasmic reticulum stress is associated with neuroprotection against apoptosis via autophagy activation in a rat model of subarachnoid hemorrhage. Neurosci Lett. 2014;563:160-5.

45. Zhang T, Wu P, Budbazar E, et al. Mitophagy reduces oxidative stress via Keap1 (Kelch-like epichlorohydrin-associated protein 1)/Nrf2 (nuclear factorE2-related factor 2)/PHB2 (prohibitin 2) pathway after subarachnoid hemorrhage in rats. Stroke. 2019;50(4):978-88.

46. Zhu Q, Enkhjargal B, Huang L, Zhang T, Sun C, Xie Z, Wu P, Mo J, Tang J, Xie Z, Zhang JH. Aggf1 attenuates neuroinflammation and BBB disruption via PI3K/Akt/NF-kB pathway after subarachnoid hemorrhage in rats. J Neuroinflammation. 2018;15(1):178.

47. Zhou K, Enkhjargal B, Xie Z, Sun C, Wu L, Malaguit J, Chen S, Tang J, Zhang J, Zhang JH. Dihydrolipoic acid inhibits lysosomal rupture and NLRP3 through lysosome-associated membrane protein-1/calcium/calmodulindependent protein kinase II/TAK1 pathways after subarachnoid hemorrhage in rat. Stroke. 2018;49(1):175-83.

48. Zhao Q, Che X, Zhang H, Fan P, Tan G, Liu L, Jiang D, Zhao J, Xiang X, Liang $Y$, Sun $X$, He Z. Thioredoxin-interacting protein links endoplasmic reticulum stress to inflammatory brain injury and apoptosis after subarachnoid hemorrhage. J Neuroinflammation. 2017;14(1):104.

49. Zhang X, Wu Q, Zhang Q, Lu Y, Liu J, Li W, Lv S, Zhou M, Zhang X, Hang C. Resveratrol attenuates early brain injury after experimental subarachnoid hemorrhage via inhibition of NLRP3 inflammasome activation. Front Neurosci. 2017a;11:611.

50. Zhang P, Yi LH, Meng GY, Zhang HY, Sun HH, Cui LQ. Apelin-13 attenuates cisplatin-induced cardiotoxicity through inhibition of ROS-mediated DNA damage and regulation of MAPKs and AKT pathways. Free Radic Res. 2017b; 51(5):449-59.

51. Zhao H, Zhang X, Dai Z, Feng Y, Li Q, Zhang JH, et al. P2X7 receptor suppression preserves blood-brain barrier through inhibiting RhoA activation after experimental intracerebral hemorrhage in rats. Sci Rep. 2016;6:23286.

52. Zhao Y, Li Q, Zhao W, Li J, Sun Y, Liu K, Liu B, Zhang N. Astragaloside IV and cycloastragenol are equally effective in inhibition of endoplasmic reticulum stress-associated TXNIP/NLRP3 inflammasome activation in the endothelium. J Ethnopharmacol. 2015;169:210-8.

53. Zhang KZ, Kaufman RJ. From endoplasmic-reticulum stress to the inflammatory response. Nature. 2008;454:455-62.

\section{Publisher's Note}

Springer Nature remains neutral with regard to jurisdictional claims in published maps and institutional affiliations. 